\title{
NMR Structure of Citrobacter freundii AmpD, Comparison with Bacteriophage T7 Lysozyme and Homology with PGRP Domains
}

\author{
Edvards Liepinsh', Catherine Généreux², Dominique Dehareng ${ }^{2}$ \\ Bernard Joris ${ }^{2}$ and Gottfried Otting ${ }^{1,3 *}$
}

${ }^{1}$ Department of Medical

Biochemistry and Biophysics

Karolinska Institute, S-17177

Stockholm, Sweden

${ }^{2}$ Center for Protein Engineering Liège University, Institut de Chimie, B6, Sart-Tilman

B-4000 Liège, Belgium

${ }^{3}$ Research School of Chemistry Australian National University Canberra ACT 0200, Australia

\begin{abstract}
AmpD is a bacterial amidase involved in the recycling of cell-wall fragments in Gram-negative bacteria. Inactivation of AmpD leads to derepression of $\beta$-lactamase expression, presenting a major pathway for the acquisition of constitutive antibiotic resistance. Here, we report the NMR structure of AmpD from Citrobacter freundii (PDB accession code 1J3G). A deep substrate-binding pocket explains the observed specificity for low molecular mass substrates. The fold is related to that of bacteriophage T7 lysozyme. Both proteins bind zinc at a conserved site and require zinc for amidase activity, although the enzymatic mechanism seems to differ in detail. The structure-based sequence alignment identifies conserved features that are also conserved in the eukaryotic peptidoglycan recognition protein (PGRP) domains, including the zinc-coordination site in several of them. PGRP domains thus belong to the same fold family and, where zinc-binding residues are conserved, may have amidase activity. This hypothesis is supported by the observation that human serum $\mathrm{N}$-acetylmuramyl-L-alanine amidase seems to be identical with a soluble form of human PGRP-L.
\end{abstract}

(C) 2003 Published by Elsevier Science Ltd recognition protein domain; zinc amidase

\section{Introduction}

Peptidoglycan, the major constituent of bacterial cell walls, confers rigidity, shape and resistance against osmotic lysis. The exceptional stability of this macromolecule results from peptide crosslinks between the glycan chains composed of alternating units of $N$-acetyl-glucosamine (GlcNAc) and substituted $N$-acetyl muramic acid (MurNAc) residues. In Gram-negative bacteria, MurNAc residues are derivatised by L-alanyl- $\gamma$-D-glutamyl-

E. L. and C. G. contributed equally to this work.

Abbreviations used: GlcNAc, $N$-acetyl-glucosamine; ICPMS, inductively coupled plasma mass spectrometry; MA, human serum $\mathrm{N}$-acetylmuramyl-L-alanine amidase; MurNAc, $N$-acetyl muramic acid; NMR, nuclear magnetic resonance; NOE, nuclear Overhauser effect; NOESY, two-dimensional NOE spectroscopy; r.m.s.d., root-mean-square deviation; PGRP, peptidoglycan recognition protein; TOCSY, total correlation spectroscopy.

E-mail address of the corresponding author: gottfried.otting@anu.edu.au
meso-diaminopimelyl-D-alanine (L-Ala-D-Glu-m$\mathrm{A}_{2}$ pm-D-Ala) linked to the sugar via an amide bond between the L-Ala amino group and the carboxyl group of the muramyl residue. Crosslinks between two adjacent glycan chains form between $m-\mathrm{A}_{2} \mathrm{pm}$ in one peptide and D-Ala in another.

Cell growth and division require continuous peptidoglycan remodelling by peptidoglycan hydrolases. In Escherichia coli and, probably, most other Gram-negative bacteria, $40-50 \%$ of the peptidoglycan is degraded during each generation by autolytic enzymes and $90 \%$ of the degraded products are recycled. ${ }^{1-3} \mathrm{AmpD}$ is a cytosolic amidase (EC 3.5.1.28) involved in this recycling. AmpD specifically cleaves the amide bond between the lactyl group of $\mathrm{N}$-acetylmuramic acid and the $\alpha$-amino group of the L-alanine in degradation products containing an anhydro $N$-acetylmuramyl moiety, such as 1,6-anhydroMurNAc-tripeptides, -tetrapeptides and GlcNAc1,6-anhydroMurNAc-tri- and -tetrapeptides. In contrast, cytoplasmic precursors of peptidoglycan, 
such as UDP-MurNAc-tripeptide and UDPMurNAc-pentapeptide, are not hydrolyzed by AmpD. ${ }^{4,5}$

Importantly, high intracellular levels of uncleaved 1,6-anhydroMurNAc-tripeptides allosterically induce the transcriptional activator AmpR, resulting in derepression of $\beta$-lactamase expression in bacteria with inducible $\beta$ lactamases. ${ }^{4}$ As AmpD activity is not essential for bacteria, inactivating mutations of AmpD present a straightforward mechanism by which bacteria can gain constitutive $\beta$-lactam resistance. In fact, the high-volume use of third-generation cephalosporins has resulted in the selection of constitutively $\beta$-lactam resistant $\mathrm{AmpD}^{-}$populations of Enterobacter, Serratia, Morganella, Citrobacter, Pseudomonas and Providencia. These present a serious problem in hospitals and intensive care units, where resistance levels against third-generation cephalosporins above $40 \%$ were reported for, e.g. Enterobacter cloacae. ${ }^{6}$

To date, over 50 bacterial amidases have been identified by genomic sequencing and classified as presumable $\mathrm{N}$-acetylmuramyl-L-alanine amidases, including functional homologues of AmpD as well as more general cell-wall hydrolases. The only 3D structure determined for any member of this class of enzymes is the crystal structure of bacteriophage T7 lysozyme, ${ }^{7,8}$ which displays only about $20 \%$ sequence identity with AmpDs. Here, we report the 3D structure of AmpD from Citrobacter freundii, which is the biochemically best characterised AmpD and almost $90 \%$ identical in sequence with Escherichia coli AmpD, with most differences confined to the $\mathrm{N}$ and $C$ termini. AmpD is shown to require zinc for enzymatic activity and a model of the enzyme-substrate complex is established to explain its substrate specificity. Structure-based sequence alignment of AmpD and T7 lysozyme indicates that peptidoglycan recognition protein (PGRP) domains have the same fold and substrate-binding site, including zinc-binding residues. This suggests hydrolytic activity for several of the PGRP domains, although no enzymatic activity has been found for the PGRP domains characterised to date. PGRP domains represent the only group of eukaryotic proteins known with significant sequence homology to AmpD and T7 lysozyme. They attracted much current interest, since some of them were shown to trigger immune responses by recognition of bacterial cell walls. ${ }^{9-13}$

\section{Results and Discussion}

\section{Solution structure of $C$. freundii AmpD and comparison to $\mathrm{T7}$ lysozyme}

The fold of AmpD comprises three $\beta$-sheets, four $\alpha$-helices, and one $33_{10}$-helical turn (Figure $1(\mathrm{a}))$. They include residues $3-5\left(\beta_{1}\right), 7-8\left(\beta_{2}\right)$, $12-14\left(\beta_{3}\right), 31-33\left(\beta_{4}\right), 46-53\left(\alpha_{1}\right), 62-69\left(\alpha_{2}\right)$, 76-79 $\left(\beta_{5}\right), 83-87\left(\beta_{6}\right), 100-102\left(\beta_{7}\right), 105-107$ $\left(\beta_{8}\right), 115-117\left(\beta_{9}\right), 127-143\left(\alpha_{3}\right), 147-149\left(3_{10}\right)$, $151-152\left(\beta_{10}\right)$ and $171-177\left(\alpha_{4}\right)$, as identified by the Kabsch-Sander algorithm in the majority of the NMR conformers. ${ }^{14}$ A search of the protein data bank (PDB) with the program DALI ${ }^{15}$ revealed T7 lysozyme ${ }^{7}$ as the only structural relative, with an r.m.s.d. of $3.3 \AA$ for 120 aligned $C^{\alpha}$ atoms. The structural conservation is most apparent for strands $\beta_{4}, \beta_{5}, \beta_{6}, \beta_{9}$, and $\beta_{10}$ in AmpD, which form a mostly parallel $\beta$-sheet of the same topology as in T7 lysozyme. In AmpD, however, this sheet is extended by a sixth strand, $\beta_{3}$ (Figure $1(a)$ and (b)). Similarly, helices 1, 3 and 4 are conserved between AmpD and T7 lysozyme, while $\alpha_{2}$ and the $3_{10}$-helical turn have no counterpart in $\mathrm{T} 7$ lysozyme. In addition, AmpD contains two sheets composed of two antiparallel $\beta$-strands each $\left(\beta_{1} / \beta_{2}\right.$ and $\beta_{7} / \beta_{8}$, respectively) that are not present in the T7 lysozyme structure.

All peptide bonds are in the trans conformation. In particular, the trans conformation was assumed for Pro165, although the corresponding residue in T7 lysozyme, Pro131, forms a cis-peptide bond. This was based on the observation of a weak sequential $\mathrm{H}^{\alpha}-\mathrm{C}^{\delta} \mathrm{H}_{2} \mathrm{NOE}$. The presence or absence of a sequential $\mathrm{H}^{\alpha}-\mathrm{H}^{\alpha} \mathrm{NOE}$, expected for a cispeptide bond, could not be verified due to chemical shift degeneracy. As the sequence alignment shows insertions and deletions nearby (Figure 2), the conformation of this proline residue is not necessarily conserved.

Inductively coupled plasma mass spectrometry (ICPMS) showed that AmpD binds a single divalent zinc ion. In the NMR structure, this $\mathrm{Zn}^{2+}$ is bound by residues homologous to the $\mathrm{Zn}^{2+}$-binding residues of T7 lysozyme (Figure 2). Like T7 lysozyme, AmpD is inactivated by metal chelators (C. G. \& B. J., unpublished results).

The ${ }^{15} \mathrm{~N}$-chemical shifts of all eight histidine side-chains were assigned and indicated that the side-chains of histidines $34,61,75,96$ and 154 are uncharged at $\mathrm{pH} 5.5\left({ }^{15} \mathrm{~N}\right.$ chemical shifts at about $250 \mathrm{ppm}$ and $220 \mathrm{ppm}$ for free and $\mathrm{Zn}^{2+}$ coordinating nitrogen atoms, respectively) with hydrogen atoms only on $\mathrm{N}^{\epsilon 2}$, while the sidechains of histidines 18, 69 and 183 were protonated $\left({ }^{15} \mathrm{~N}\right.$ chemical shifts below $\left.200 \mathrm{ppm}\right) \cdot{ }^{16}$ His75 and His96 are completely buried like the corresponding histidine residues in T7 lysozyme. The chemical shifts indicate that His34 and His154 coordinate $\mathrm{Zn}^{2+}$ via their $\mathrm{N}^{\delta 1}$ atom, in complete analogy to T7 lysozyme. His61 at the amino-terminal end of helix $\alpha_{2}$ is solvent-exposed yet unprotonated, presumably due to the dipolar field of this helix. In analogy to T7 lysozyme, where Cys130 represents the third zinc coordinating residue, Asp164 was assumed to coordinate the zinc ion in AmpD. This assumption is supported by the fact that the Asp164Glu and Asp164Ala mutants are enzymatically inactive (C. G. \& B. J., unpublished results). ${ }^{17}$ 
(a)

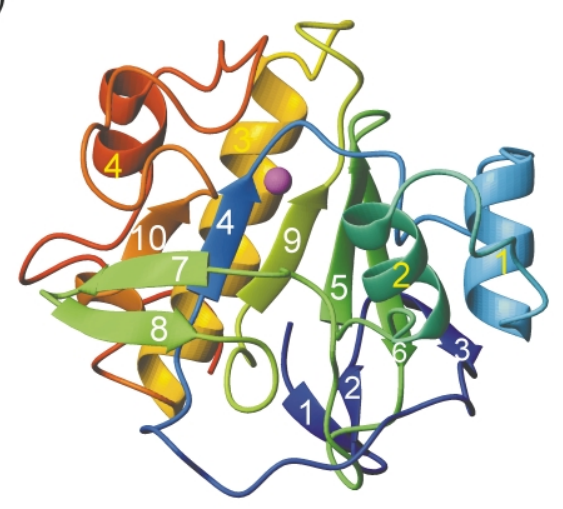

(c)

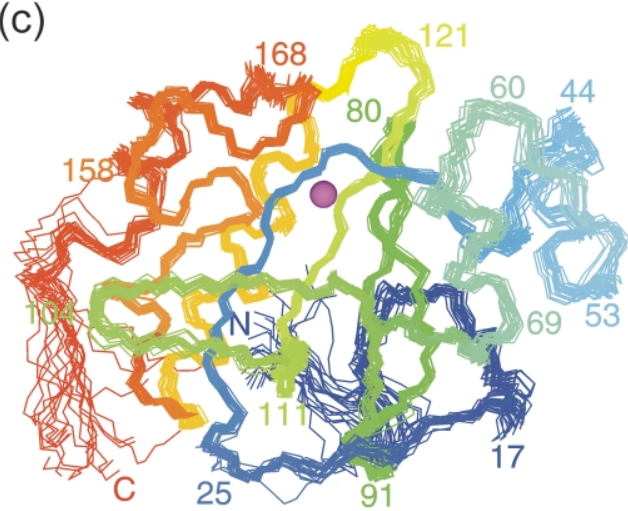

(d)

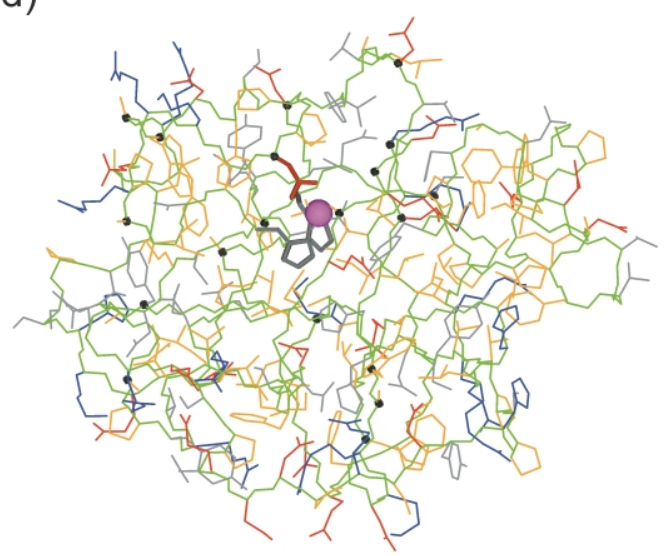

(b)

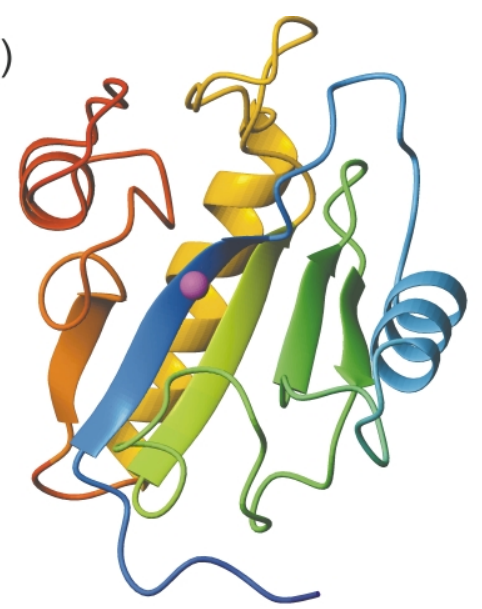

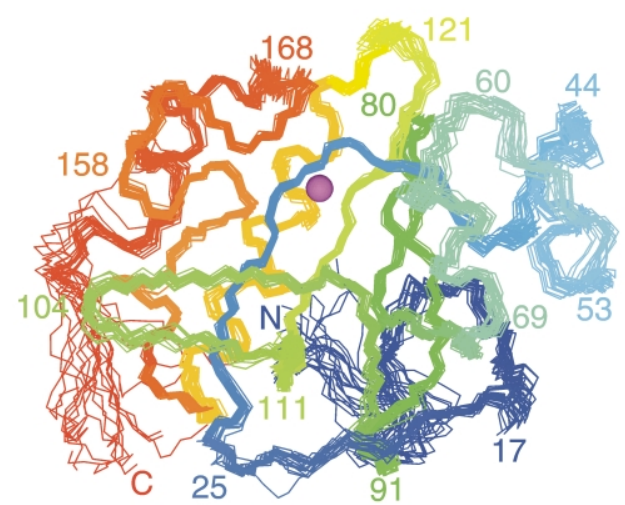

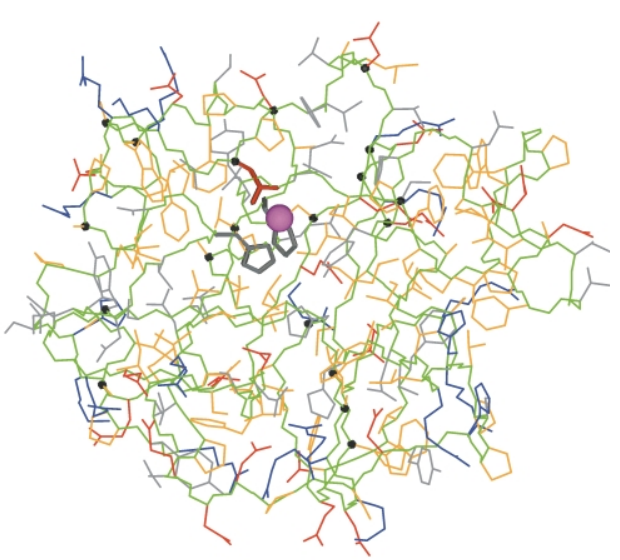

Figure 1. Solution structure of AmpD and comparison with T7 lysozyme. (a) Ribbon representation of AmpD. The $\beta$-strands and $\alpha$-helices are numbered as in Figure 2. White and yellow numbers distinguish strands and helices, respectively. The zinc ion is shown in purple. (b) Ribbon representation of T7 lysozyme (PDB code 1LBA). Secondary-structure elements homologous to AmpD are coloured as in (a). (c) Stereo view of AmpD, showing a superposition of the backbone atoms in the 20 conformers representing the NMR structure (Table 1), in the same orientation as in (a). Numbers identify sequence positions. (d) Stereo view of the AmpD conformer closest to the mean structure of the 20 conformers shown in (c), using a heavy-atom representation in the same orientation as in (c). The polypeptide backbone is drawn in green. The following colors were used for the side-chains: blue, Arg, Lys, positively charged His; red, Glu, Asp; yellow, Ala, Cys, Ile, Leu, Met, Phe, Pro, Trp, Val; gray, Asn, Gln, Ser, Thr, Tyr, uncharged His. The zinc ion is shown as a purple sphere, with the side-chains of the coordinating residues His34, His154 and Asp164 drawn in bold. Black spheres mark the $\mathrm{C}^{\alpha}$ positions of those residues, where point mutations have been found in constituitively $\beta$-lactamase expressing bacteria. ${ }^{17}$ 


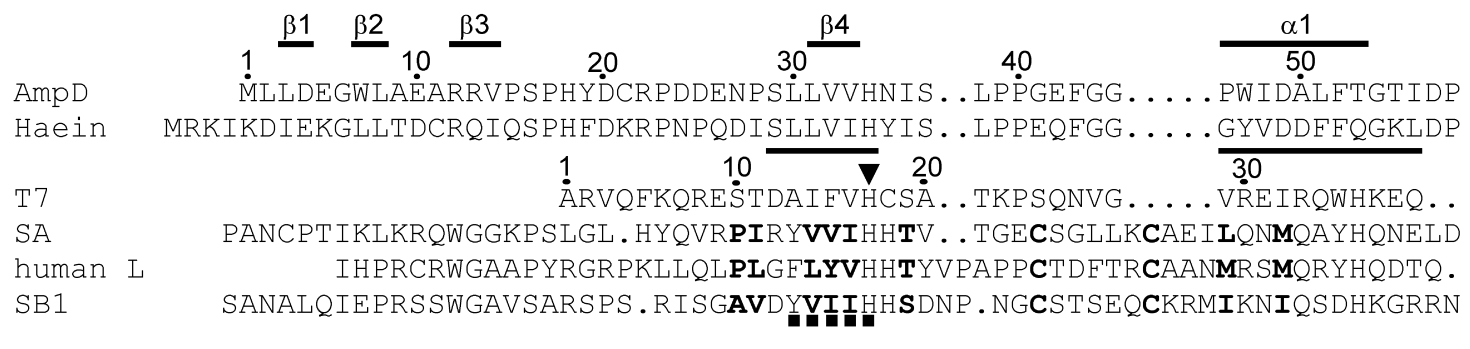

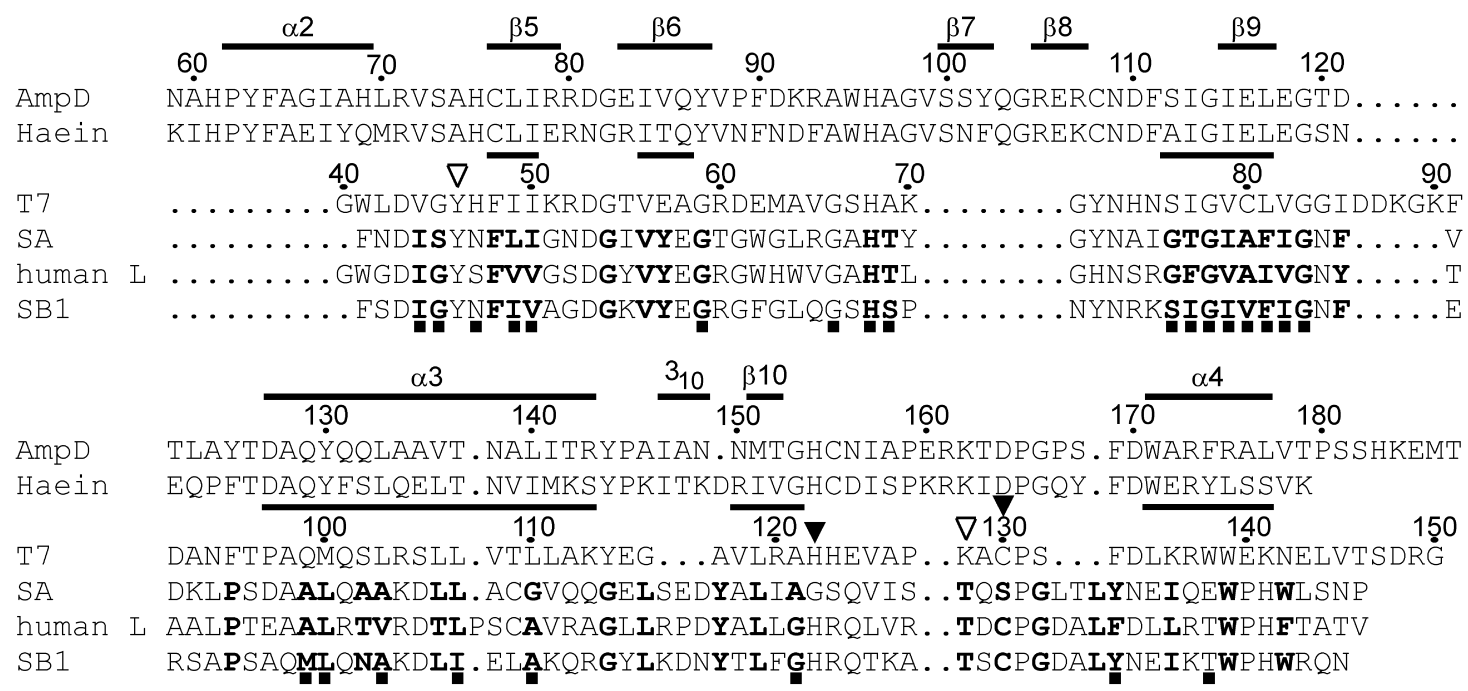

Figure 2. Multiple alignment of the amino acid sequences of Citrobacter freundii AmpD (AmpD), Haemophilus influenzae AmpD (Haein), bacteriophage T7 lysozyme (T7) and peptidoglycan recognition domains from Drosophila (SA and SB1) and human (human L). Sequence numbers are shown for AmpD and T7 lysozyme. Bars indicate the locations of helices and $\beta$-strands in AmpD and T7 lysozyme, respectively. Filled triangles identify $\mathrm{Zn}^{2+}$-coordinating residues in T7 lysozyme and AmpD. Open triangles identify catalytically important residues in T7 lysozyme. ${ }^{7}$ Bold characters in the three PGRP domains highlight residues that are uncharged (including His) in all 26 untruncated PGRP domain sequences reported by Werner et al. and Liu et al. ${ }^{13,19}$ Filled squares identify residues with a side-chain solvent-accessibility of $<10 \%$ in both AmpD and T7 lysozyme.

\section{Interaction with a substrate analogue and model of the enzyme-substrate complex}

While no high-affinity inhibitor of AmpD is known, MurNAc-L-Ala-D-Gln (Bachem, Switzerland) displayed an inhibitory effect in enzymatic assays (C. G. \& B. J., unpublished results). ${ }^{15} \mathrm{~N}-$ HSQC spectra recorded for ${ }^{15} \mathrm{~N}$-labelled AmpD in the presence of a 100-fold excess of this substrate analogue, however, did not reveal any significant shift of amide resonances, indicating that any binding interaction must be weak. Yet, significant line broadening was observed for some of the resonances, most significantly the side-chain $\mathrm{H}^{\mathrm{N}}$ of the Zn-coordinating residue His154, which broadened beyond detection (data not shown). No broadening could be detected for any of the other histidine residues, but a line broadening by ca $30 \%$ was observed for the Zn-coordinating residue Asp164. Furthermore, the amide resonance of Tyr63 was broadened about twofold. These data strongly suggest that the $\mathrm{Zn}^{2+}$ marks the location of the active site. Further small line broadenings were observed for the amide groups of Lys162, Arg22, Tyr19, and Leu70, which could be due to interactions with the peptide segment of the substrate analogue.

In order to check whether the site with the $\mathrm{Zn}^{2+}$ could accommodate a substrate molecule and hence mark the active site, one conformer of the ensemble of NMR structures was selected to model the enzyme-substrate complex (Figure 3). As AmpD positively discriminates anhydromuramic acid versus muramic acid derivatives, the carbohydrate moiety was assumed to be in intimate contact with the enzyme. The model shows that the carbonyl oxygen atom of the scissile amide bond can be placed in close proximity to the $\mathrm{Zn}^{2+}$, with the anhydroMurNAc moiety residing in a recognition pocket. The accuracy of the model is insufficient to pinpoint specific intermolecular interactions that would explain the preference of AmpD for anhydroMurNAc over MurNAc. However, the availability of a recognition pocket of limited size would explain the specificity of AmpD for small substrate molecules. The pocket appears to be sufficiently deep that it 


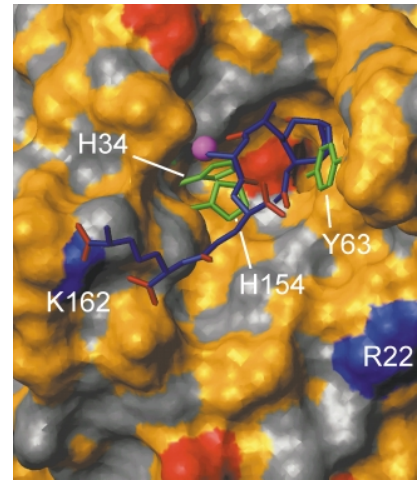

Figure 3. Model of the AmpD-substrate complex. The solvent-accessible surface is shown with the following color code for different chemical groups: yellow, hydrophobic; gray, hydrophilic; red, negatively charged; blue, positively charged. The zinc atom is displayed as a purple sphere. The side-chains of the $\mathrm{Zn}^{2+}$-coordinating residues and of Tyr63 were excluded from the surface calculation and drawn with green lines instead. The substrate, 1,6-anhydro MurNAc-L-alanyl- $\gamma$-D-glutamyl-mesodiaminopimelic acid, is drawn in a line representation (blue) with carbonyl and carboxyl bonds in red.

could accommodate a GlcNAc-1,6-anhydroMurNAc moiety, but not a larger carbohydrate unit or the anabolic compound UDP-MurNAc-pentapeptide. The existence of this pocket is determined largely by the loop connecting strand 4 and helix 1 , which assumes a very different conformation in AmpD and T7 lysozyme (Figure 1(a) and (b)), although there is no insertion or deletion in this amino-acid segment (Figure 2). The structural difference between AmpD and T7 lysozyme in this region is significant, as the conformation of this loop is conserved between free ${ }^{7}$ and polymerase-complexed ${ }^{8}$ T7 lysozyme, and it appears to be well defined in AmpD. The different loop conformation, together with the additional strands $\beta_{7}$ and $\beta_{8}$ (Figure 1(a) and (b)), is probably an important determinant of the specificity of AmpD for small substrate molecules, while T7 lysozyme is able to act on polymeric substrates.

The model predicts only superficial attachment of the peptide part to the protein surface, explaining the weak discrimination between peptide moieties of different lengths. ${ }^{5}$ In Figure 3, the terminal end of the peptide was modeled to contact Lys162, as mutations at this site reduced enzymatic activity (C. G. \& B. J., unpublished results). However, the NMR data with the substrate analogue suggest that the terminal end could also contact Arg22 and nearby residues (Figure 3). There is thus no evidence for particular specificity for meso-diaminopimelic acid in the peptide segment, although this amino acid is characteristic of Gram-negative bacteria with a cell-wall recycling system.

Besides the three zinc-coordinating residues and Lys128, Tyr46 had been identified as a catalytically important residue in T7 lysozyme (Figure 2). Its side-chain oxygen atom is about $5.3 \AA$ from the zinc ion and mutation to phenylalanine completely abrogates enzymatic activity. ${ }^{7}$ In AmpD, Tyr63 provides the closest hydroxyl group, which is located at a slightly longer distance in most of the NMR conformers (Figure 3). If residues 40-46 of T7 lysozyme were left-shifted in the sequence alignment of Figure 2 by 11 residues, Tyr63 in AmpD would align with Tyr46 in T7 lysozyme. Such an alignment is, however, not supported by the structures. Tyr63 in AmpD is located in a helix that has no counterpart in T7 lysozyme (Figure 2) and approaches the active site from a different angle. Furthermore, mutation to phenylalanine retains $10 \%$ of the enzymatic activity (C. G. \& B. J., unpublished results). Clearly, both enzymes seem to use the $\mathrm{Zn}^{2+}$ to polarise the scissile bond of the substrate, but the enzymatic mechanism seems to differ in detail.

\section{AmpD $^{-}$mutations}

AmpDs from different bacteria are highly homologous, such as the AmpD from Haemophilus influenzae (Figure 2), and their structures must be very similar to that of C. freundii AmpD. Therefore, point mutations that inactivate the AmpD from one bacterial species are probably also deleterious in C. freundii AmpD. A recent compilation of inactivation mutations from nine enterobacteria identified residues $1,7,33,37,80,82,83,94,95,102$, $117,121,127,134,146,158,164,171$ and 172 as sites of inactivating point-mutations capable of triggering constitutive $\beta$-lactamase expression. ${ }^{17}$ The spatial distribution of these residues does not highlight the location of the active site (Figure $1(\mathrm{~d})$ ), except that the mutation frequency of the active-site residue Asp164 was outstanding. ${ }^{17}$ For most of the mutated residues, the side-chain solvent exposure is less than $25 \%$, i.e. their mutation could plausibly destabilise the protein. Residues 1 , 121, 127, 158 and 172 are, however, highly solvent exposed. Mutation of Met1 presumably interferes with protein expression. Asp121 is within $10 \AA$ of the active site. The Asp127Gly mutation breaks a salt-bridge with Lys173. Inactivation by the mutations Ala158Glu (reported by Stapleton et al. ${ }^{18}$ ) and Ala172Leu, however, seems to require some unknown biological rather than structural explanation.

\section{Amidase activity in PGRP domains}

The delineation of conserved secondary structure elements between AmpD and T7 lysozyme allows an improved alignment with the PGRP domains. A total of 26 different full-length PGRP domains have been reported by Werner et al. and Liu et al. ${ }^{13,19}$ Structural conservation is suggested strongly by the observation that residues for which the side-chains are buried in both AmpD and T7 lysozyme tend to be conserved 
hydrophobic and uncharged residues in PGRP domains (Figure 2). On the basis of the number of insertions and deletions in the alignment, PGRP domains are related to T7 lysozyme more closely than to AmpD. For example, they lack the sheet composed of strands $\beta_{7}$ and $\beta_{8}$. The absence of this sheet correlates with the ability of T7 lysozyme and PGRPs to bind macromolecular rather than low molecular mass substrates. On the other hand, they seem to be more like AmpD in the loop between strand $\beta_{9}$ and helix $\alpha_{3}$ (Figure 2). Even helix $\alpha_{4}$ seems to be present in PGRP domains, as indicated by the periodical pattern of consistently uncharged residues. This pattern is, however, outof-frame compared to AmpD and T7 lysozyme, suggesting a different orientation with respect to the protein core.

A previously published alignment of PGRP domains with bacteriophage T3 lysozyme (which is $97 \%$ identical in sequence with T7 lysozyme) suggested the absence of conserved $\mathrm{Zn}^{2+}$-binding residues, ${ }^{11}$ but the sequence similarity was so low that its significance is not universally accepted. ${ }^{19}$ Our new, improved alignment, however, clearly reveals conserved $\mathrm{Zn}^{2+}$-binding residues in the soluble Drosophila SB1 protein, in the membraneattached human L protein and in several other PGRPs shown in the alignments by Werner et al. and Liu et al. ${ }^{13,19}$ In contrast, these $\mathrm{Zn}^{2+}$-binding residues are not conserved in the Drosophila SA protein (Figure 2) or in numerous other PGRPs (not shown), which thus seem to be devoid of a metal-binding site. Interestingly, this group of PGRPs seems capable of binding to carbohydrates and exerting antibacterial activity in a peptidoglycan and zinc-independent way. ${ }^{20-22}$

All PGRP domains with a conserved $\mathrm{Zn}^{2+}$-binding site (six of 26 different PGRP domains) also have a conserved tyrosine residue ${ }^{13,19}$ that corresponds to the catalytically critical residue Tyr46 in T7 lysozyme, and a histidine residue corresponding to His96 in AmpD that is buried near the active site. In T7 lysozyme, Lys128 is the only residue besides Tyr46 that has been shown to be catalytically important. It is also conserved in AmpD (Lys162), although it is not one of the zinc-coordinating residues. The PGRP domains with putative zinc-binding site all have threonine at this position. In T7 lysozyme, the Lys128Thr mutant reduces amidase activity but does not fully inactivate. ${ }^{7}$

There is incidental evidence for amidase activity by at least one member of the PGRP family, as a soluble form of human PGRP-L $\mathrm{L}^{19}$ (denoted human $\mathrm{L}$ in Figure 2) seems to be identical with the previously characterised serum $\mathrm{N}$-acetylmuramyl-Lalanine amidase (MA). ${ }^{23}$ The sequence of the 15 $\mathrm{N}$-terminal residues of MA determined by chemical microsequencing ${ }^{23}$ corresponds exactly to that of human PGRP-L, devoid of its first 21 residues. No other protein in the human genome matches this amino acid sequence. MA is an abundant, glycosylated, dimeric enzyme with a molecular mass of $73 \mathrm{kDa}$ per monomeric subunit. Considering the increase in molecular mass due to glycosylation, this is in good agreement with the molecular mass of human PGRP-L calculated from its amino acid sequence (60 kDa for residues 22-576). MA was reported to require high concentrations of $\mathrm{MgCl}_{2}(10 \mathrm{mM})$ for optimum substrate binding and enzymatic activity, while $\mathrm{Zn}^{2+}$-dependence was not tested. ${ }^{23}$ In view of our results and the fact that physiological concentrations of $\mathrm{Mg}^{2+}$ in blood are only $0.8-1.2 \mathrm{mM}$, this enzyme may work better with $\mathrm{Zn}^{2+}$ than with $\mathrm{Mg}^{2+}$.

In conclusion, amidase activity is highly plausible for all PGRP domains that are capable of binding $\mathrm{Zn}^{2+}$. The structural homologies established here will allow the informed design of active-site mutations in PGRP domains that interfere with function without disrupting their structure. It is remarkable how, in the course of evolution, a classical bacterial household enzyme has been recruited and turned against bacteria by bacteriophages and by the eukaryotic immune system.

\section{Materials and Methods}

\section{AmpD expression and purification}

For the preparation of isotope-labelled samples, the gene of $C$. freundii AmpD was cloned into pET-9a and transformed into E. coli BL21(DE3)/pLysS cells (Invitrogen), where AmpD expressed as a cytoplasmic protein. Uniformly ${ }^{15} \mathrm{~N}$ and ${ }^{15} \mathrm{~N} /{ }^{13} \mathrm{C}$-labelled protein samples were prepared overnight at $37^{\circ} \mathrm{C}$ in $100 \mathrm{ml}$ of ${ }^{15} \mathrm{~N}$ Celtone (Martek Biosciences Corporation) or ${ }^{15} \mathrm{~N} /{ }^{13} \mathrm{C}$ Celtone broth supplemented with $50 \mu \mathrm{g} \mathrm{ml}^{-1}$ kanamycin and $30 \mu \mathrm{g} \mathrm{ml}^{-1}$ chloramphenicol. These cultures were used to inoculate 51 of M9 minimal medium, containing $0.05 \%(\mathrm{w} / \mathrm{v})$ thiamine, $0.001 \%(\mathrm{w} / \mathrm{v})$ biotin, $0.01 \%$ $(\mathrm{w} / \mathrm{v})$ choline chloride, $0.001 \%(\mathrm{w} / \mathrm{v})$ folic acid, $0.01 \%$ $(\mathrm{w} / \mathrm{v})$ niacinamide, $0.002 \%(\mathrm{w} / \mathrm{v})$ pyridoxal, $0.001 \%$ (w/v) riboflavin, $4 \mathrm{mM} \mathrm{MgSO} 4,13 \mu \mathrm{M} \mathrm{FeSO}, 20 \mathrm{mM}$ ${ }^{15} \mathrm{NH}_{4} \mathrm{Cl}$ and glucose $(30 \mathrm{mM}$ unlabelled glucose or $9 \mathrm{mM}{ }^{13} \mathrm{C}$-glucose). Similarly, a sample with $10 \%$ biosynthetic fractional ${ }^{13} \mathrm{C}$-labeling was prepared using a 1:9 mixture of ${ }^{13} \mathrm{C}$-labelled and ${ }^{12} \mathrm{C}$-labelled glucose at a total concentration of $30 \mathrm{mM}$. Growth was accomplished at $30^{\circ} \mathrm{C}$. When the cell density reached an $A_{600}$ value of 1, IPTG (1 mM final concentration) was added and growth continued overnight.

After induction, the cells were harvested by centrifugation $\left(8300 \mathrm{~g}, 15\right.$ minutes, $4^{\circ} \mathrm{C}$ ), washed with $150 \mathrm{mM}$ $\mathrm{NaCl}$ and resuspended in buffer A $(20 \mathrm{mM}$ phosphate buffer ( $\mathrm{pH} 7.0$ ) containing $1 \mathrm{mM}$ DTT). Cell lysis was achieved with the help of a cell disintegrator (Serie Z, Constant System, Warwick, UK) at $4{ }^{\circ} \mathrm{C}$ with a pressure of $1.9 \mathrm{kbar}\left(1 \mathrm{bar}=10^{5} \mathrm{~Pa}\right)$. Benzonase ( 5 units/litre of culture) and Pefabloc $(0.1 \mathrm{mg} / \mathrm{ml}$, final concentration) were added to cell lysates. The insoluble material was removed by centrifugation at $20,000 \mathrm{~g}$ for 15 minutes at $4{ }^{\circ} \mathrm{C}$. The supernatant was dialyzed overnight against buffer A. After dialysis, the lysate was centrifuged at $20,000 \mathrm{~g}$ for 15 minutes at $4{ }^{\circ} \mathrm{C}$, microfiltered on $0.70 \mu \mathrm{m}$ and $0.45 \mu \mathrm{m}$ Millipore filters and loaded onto a Q-Sepharose column $(2.6 \mathrm{~cm} \times 30 \mathrm{~cm}, 160 \mathrm{ml}$, Amersham Pharmacia Biotech, Uppsala, Sweden) equilibrated in buffer A. The column was eluted with a linear gradient of $\mathrm{NaCl}(720 \mathrm{ml})$ from $0 \mathrm{M}$ to $1 \mathrm{M}$. The peak fractions 


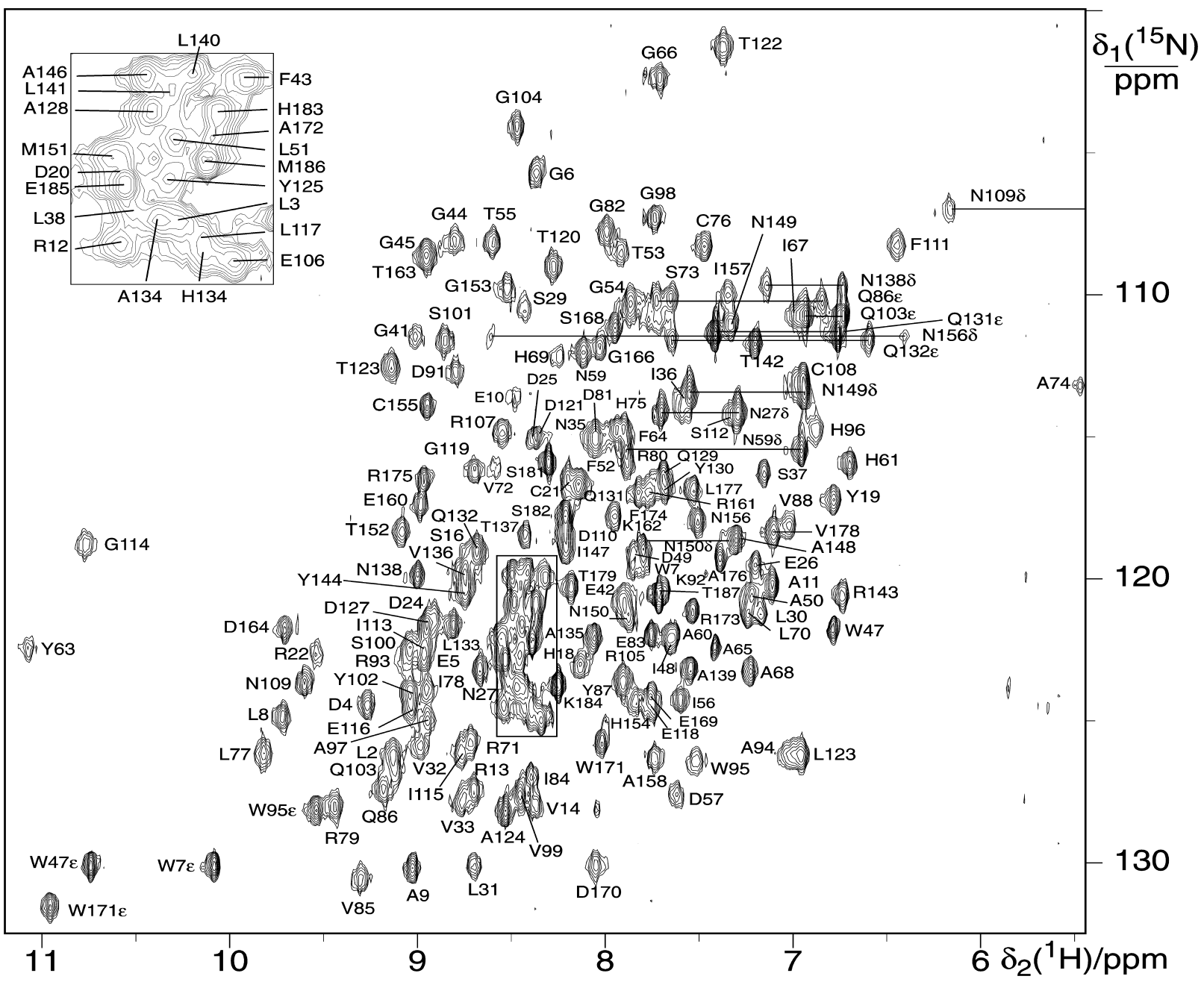

Figure 4. ${ }^{15} \mathrm{~N}-\mathrm{HSQC}$ spectrum of $\mathrm{C}$. freundii AmpD. The spectrum was recorded using an $0.3 \mathrm{mM}$ sample of uniformly ${ }^{15} \mathrm{~N}$-labelled AmpD at pH 5.5 and $31{ }^{\circ} \mathrm{C}$ in the presence of $2 \mathrm{mM} \mathrm{ZnSO}_{4}$ at $600 \mathrm{MHz}{ }^{1} \mathrm{H}$ NMR frequency. Backbone resonances are assigned with the amino acid codes and sequence numbers. Side-chain resonances are labelled in addition with the greek letters of the respective side-chains. The second peak of the $\mathrm{N}^{\delta} \mathrm{H}_{2}$ group of Asn109 is outside the plot region. The inset is an expansion of the spectral region identified by a box.

containing the AmpD amidase were pooled, concentrated to $10 \mathrm{ml}$ by ultrafiltration on a $5000 \mathrm{Da}$ cutoff Amicon membrane and applied onto a molecular sieve column of Sephacryl S100 HR $(2.6 \mathrm{~cm} \times 95 \mathrm{~cm}, 500 \mathrm{ml}$, Amersham Pharmacia Biotech) equilibrated in buffer A. The fractions containing the amidase were collected and concentrated. After dialysis against $1.6 \mathrm{M}$ $\left(\mathrm{NH}_{4}\right)_{2} \mathrm{SO}_{4}$, the enzyme was purified further by a Source 15 ISO column $(1.6 \mathrm{~cm} \times 10 \mathrm{~cm}, 20 \mathrm{ml}$, Amersham Pharmacia Biotech), eluting with a linear gradient from $1.5 \mathrm{M}$ to $0 \mathrm{M}\left(\mathrm{NH}_{4}\right)_{2} \mathrm{SO}_{4}$. The fractions containing AmpD were collected and dialyzed against buffer and concentrated by ultrafiltration. After addition of $\mathrm{NaN}_{3}$ ( $1 \mathrm{mM}$, final concentration), the enzyme was stored at $4{ }^{\circ} \mathrm{C}$. The protein content of the column fractions was monitored by SDS-PAGE. The concentration of AmpD was determined from its absorbance at $280 \mathrm{~nm}$, using an extinction coefficient of $31,960 \mathrm{M}^{-1} \mathrm{~cm}^{-1}$. Typical yields were $12-18 \mathrm{mg}$ of pure protein/litre of medium for unlabelled AmpD and $2-8 \mathrm{mg} / 1$ for isotope-labelled AmpD.

\section{Metal content analysis}

Pure protein $(1 \mathrm{ml}$ of $48 \mu \mathrm{M})$ was dialysed overnight against $250 \mathrm{ml}$ of $10 \mathrm{mM}$ Hepes (pH 7.0) in doubly distilled water. The zinc content of the enzyme was measured by ICPMS.

\section{NMR spectroscopy}

NMR spectra were recorded at $31^{\circ} \mathrm{C}$ at $\mathrm{pH} 5.5$ and 7.0, using $\sim 0.3 \mathrm{mM}$ solutions of AmpD in $95 \% \mathrm{H}_{2} \mathrm{O} / 5 \%$ ${ }^{2} \mathrm{H}_{2} \mathrm{O}$ or $100 \%{ }^{2} \mathrm{H}_{2} \mathrm{O}$ at a ${ }^{1} \mathrm{H}$ NMR frequency of $600 \mathrm{MHz}$ on a Bruker DMX 600 NMR spectrometer. Samples were prone to irreversible precipitation. The sample stability at $\mathrm{pH}$ values below 7 was significantly improved by the addition of $2 \mathrm{mM} \mathrm{ZnSO}_{4}$. Most of the NOE restraints were collected from a homonuclear NOESY spectrum recorded at $800 \mathrm{MHz}$ on a Varian Unity INOVA NMR spectrometer with a sample of unlabelled AmpD (40 ms mixing time, $t_{1 \max }=63 \mathrm{~ms}$ and $t_{2 \max }=128 \mathrm{~ms}, 3-9-19$ water suppression, one week total recording time). 
Table 1. Structural statistics for the NMR conformers

\begin{tabular}{|c|c|}
\hline Parameter & Value \\
\hline No. assigned NOE cross-peaks & 2820 \\
\hline No. non-redundant NOE upper-distance limits & 1879 \\
\hline No. scalar coupling constants ${ }^{\mathbf{a}}$ & 361 \\
\hline No. residual ${ }^{1} \mathrm{H}-{ }^{15} \mathrm{~N}$ dipolar couplings & 145 \\
\hline No. dihedral-angle restraints & 488 \\
\hline Intra-protein AMBER energy $\left(\mathrm{kcal} \mathrm{mol}^{-1}\right)$ & $-7640 \pm 90$ \\
\hline Maximum NOE-restraint violations $(\AA)$ & $0.10 \pm 0.00$ \\
\hline $\begin{array}{l}\text { Maximum dihedral-angle restraint violations } \\
\text { (deg.) }\end{array}$ & $3.3 \pm 0.4$ \\
\hline r.m.s.d. to the mean for $\mathrm{N}, \mathrm{C}^{\alpha}$ and $\mathrm{C}^{\prime}(\AA)^{\mathbf{b}}$ 。 & $0.61 \pm 0.10$ \\
\hline r.m.s.d. to the mean for all heavy-atoms $(\AA)^{\mathbf{b}}$ & $0.99 \pm 0.11$ \\
\hline \multicolumn{2}{|l|}{ Ramachandran plot appearance } \\
\hline Most favoured regions (\%) & 70.2 \\
\hline Additionally allowed regions (\%) & 28.2 \\
\hline Generously allowed regions (\%) & 1.6 \\
\hline Disallowed regions $(\%)$ & 0.1 \\
\hline $\begin{array}{l}\text { a } 146^{3} J\left(\mathrm{H}^{\mathrm{N}}-\mathrm{H}^{\alpha}\right), 215^{3} J\left(\mathrm{H}^{\alpha}-\mathrm{H}^{\beta}\right) \text {. } \\
\text { b } \text { For residues } 2-180 . \\
\text { c From PROCHECK-NMR. }{ }^{29}\end{array}$ & \\
\hline
\end{tabular}

Resonance assignments (Figure 4) were obtained from standard 3D $\mathrm{HNCO}, \mathrm{HNCA}, \mathrm{HN}(\mathrm{CO}) \mathrm{CA}, \mathrm{CBCA}(\mathrm{CO})$ $\mathrm{NH}$, NOESY- ${ }^{15} \mathrm{~N}$-heteronuclear single quantum correlation (HSQC), total correlated spectroscopy (TOCSY)${ }^{15} \mathrm{~N}-\mathrm{HSQC}$ and $\mathrm{H}(\mathrm{C}) \mathrm{CH}-\mathrm{TOCSY}$ and $\mathrm{HC}(\mathrm{CH})$-TOCSY ${ }^{24}$ experiments. Further ${ }^{1} \mathrm{H}$ resonance assignments were obtained from double quantum filtered (DQF)-COSY, clean-TOCSY (60 ms mixing time) and NOESY (40 ms and $100 \mathrm{~ms}$ mixing time) spectra recorded with unlabelled protein. Stereospecific assignments of $\mathrm{C}^{\beta} \mathrm{H}_{2}$ protons were obtained by a 3D HNHB spectrum. Stereospecific assignments of diastereotopic methyl groups were obtained from analysis of a ${ }^{13} \mathrm{C}-\mathrm{HSQC}$ spectrum of the $10 \%$ biosynthetically fractionally ${ }^{13} \mathrm{C}$-labelled sample. Side-chain ${ }^{15} \mathrm{~N}$ resonances of histidine residues were assigned using ${ }^{15} \mathrm{~N}-\mathrm{HMQC}$ spectra recorded according to Pelton et al. ${ }^{16}$ using a J-evolution delay of $17 \mathrm{~ms}$ for simultaneous detection of ${ }^{1} J_{\mathrm{NH}}$ and long-range ${ }^{2} J_{\mathrm{NH}}$ and ${ }^{3} J_{\mathrm{NH}}$ correlations. Residual dipolar couplings ${ }^{1} J_{\mathrm{NH}}$ were measured with the help of different concentrations of Pf1 phages. Coupling constants ${ }^{3} J\left(\mathrm{H}^{N}, \mathrm{H}^{\alpha}\right)$ couplings were measured by a CT-HMQC-HN experiment. ${ }^{25}$ The total recording time for all experiments was about two months. ${ }^{3} J\left(\mathrm{H}^{\alpha}, \mathrm{H}^{\beta}\right)$ couplings were estimated as 11.0 and $4.0 \mathrm{~Hz}( \pm 3.0 \mathrm{~Hz})$, respectively, when COSY, TOCSY and NOESY cross-peaks indicated the presence of large and small couplings, respectively, together with staggered conformations around the $\mathrm{C}^{\alpha}-\mathrm{C}^{\beta}$ bond.

\section{Structure calculations}

The cross-peaks in the NOESY spectra were assigned and integrated using the program XEASY. ${ }^{26}$ The NMR structure was calculated using the program DYANA, ${ }^{27}$ starting from 80 random conformers. The residue library was modified to accommodate the zinc ion. Distance constraints were introduced between $\mathrm{Zn}^{2+}$ and the coordinating atoms, which were modelled after the crystal structure of carboxypeptidase (PDB code 1QBR). The 20 conformers with the lowest residual restraint violations were energy minimised in water using the programme OPAL with standard parameters. ${ }^{28}$

Table 1 shows an overview of the restraints used and structural statistics. The Ramachandran plot was ana- lyzed using PROCHECK-NMR. ${ }^{29}$ No residue was consistently in a forbidden region of the Ramachandran in all 20 conformers.

Secondary structure elements and r.m.s.d. values were calculated using the program MOLMOL, ${ }^{30}$ which was used to create Figures 1 and 3. Side-chain solvent accessibilities were measured with a spherical probe of $1.4 \AA$ radius and calculated as a percentage of the accessibilities measured for a fully extended side-chain of residue $X$ in a helical Gly-X-Gly peptide. ${ }^{31}$ The values obtained were averaged over the 20 NMR conformers.

\section{Model building}

The geometry of the 1,6-anhydroMurNAc-tripeptide was optimised at the quantum chemistry semi-empirical AM1 level ${ }^{32}$ using the program Gaussian98 (Revision A.6, Gaussian, Pittsburgh). The start position of the complex was built empirically by docking the substrate in the cavity with the program InsightII (Biosym, San Diego). Thereafter, the geometry of the substrate complexes was optimised at the molecular mechanics level ${ }^{33}$ with the Amber force-field using the program Discover (Biosym, San Diego). The geometry optimisations were performed without water, using a dielectric constant of 2 and taking the threshold for convergence to be the maximum force fixed at $0.02 \mathrm{kcal} \mathrm{mol}^{-1} \mathrm{~A}^{-1}$.

\section{Data Bank accession codes}

The coordinates of the 20 energy-refined DYANA conformers of AmpD have been deposited in the PDB with the accession code 1J3G. The NMR chemical shifts were deposited at the BioMagResBank (BMRB) under accession code 5483 .

\section{Acknowledgements}

We thank Christine Jacobs, Johan Weigelt and Andrew Torda for initial protein samples, NMR measurements and sequence alignments, respectively, and ASLA (Riga, Latvia) for a gift of Pf1 phages. We thank Bengt Persson for searching the public and Celera human genome data bases for the MA peptide. This work was supported by the Belgian program on Interuniversity Poles of Attraction initiated by the Federal Office for Scientific, Technical and Cultural Affaires (PAI no P5/33), the Fond National de la Recherche Scientifique (FNRS, crédit aux chercheurs no 1.5201.02), the Swedish Research Council and the Swedish NMR Centre. B. J. is an FNRS associate researcher and C. G. is a fellow of the Fonds pour la Formation à la Recherche dans l'Industrie et l'Agriculture (FRIA, Brussels, Belgium). The ICPMS measurements were performed by the Laboratoire de la Santé et de l'Environnement, Institut Malvoz de la Province de Liège, Liège, Belgium. 


\section{References}

1. Goodell, E. W. (1985). Recycling of murein by Escherichia coli. J. Bacteriol. 163, 305-310.

2. Goodell, E. W. \& Schwarz, U. (1985). Release of cell wall peptides into culture medium by exponentially growing Escherichia coli. J. Bacteriol. 162, 391-397.

3. Park, J. T. (1993). Turnover and recycling of the murein sacculus in oligopeptide permease-negative strains of Escherichia coli: indirect evidence for an alternative permease system and for a monolayered sacculus. J. Bacteriol. 175, 7-11.

4. Jacobs, C., Huang, L., Bartowsky, E., Normark, S. \& Park, J. T. (1994). Bacterial cell wall recycling provides cytosolic muropeptides as effectors for $\beta$-lactamase induction. EMBO J. 13, 4684-4694.

5. Jacobs, C., Joris, B., Jamin, M., Klarsov, K., Van Beeumen, J., Mengin-Lecreulx, D. et al. (1995). AmpD, essential for both $\beta$-lactamase regulation and cell wall recycling, is a novel cytosolic $N$-acetylmuramyl-L-alanine amidase. Mol. Microbiol. 15, $553-559$.

6. Jones, R. N. (1998). Important and emerging $\beta$-lactamase mediated resistances in hospital-based pathogens: the AmpC enzymes. Diag. Microbiol. Infect. Dis. 31, 461-466.

7. Cheng, X., Zhang, X., Pflugrath, J. W. \& Studier, F. W. (1994). The structure of bacteriophage T7 lysozyme, a zinc amidase and an inhibitor of T7 RNA polymerase. Proc. Natl Acad. Sci. 91, 4034-4038.

8. Jeruzalmi, D. \& Steitz, T. A. (1998). Structure of T7 RNA polymerase complexed to the transcriptional inhibitor T7 lysozyme. EMBO J. 17, 4101-4113.

9. Choe, K., Werner, T., Stöven, S., Hultmark, D. \& Anderson, K. V. (2002). Requirement for a peptidoglycan recognition protein (PGRP) in relish activation and antibacterial immune responses in Drosophila. Science, 296, 359-362.

10. Gottar, M., Gobert, V., Michel, T., Belvin, M., Duyk, G., Hoffmann, J. A., Ferrandon, D. \& Royet, J. (2002). The Drosophila immune response against Gram-negative bacteria is mediated by a peptidoglycan recognition protein. Nature, 416, 640-644.

11. Kang, D., Liu, G., Lundström, A., Gelius, E. \& Steiner, H. (1998). A peptidoglycan recognition protein in innate immunity conserved from insects to humans. Proc. Natl Acad. Sci. USA, 95, 10078-10082.

12. Michel, T., Reichart, J. M., Hoffmann, J. A. \& Royet, J. (2001). Drosophila Toll is activated by Gram-positive bacteria through a circulating peptidoglycan recognition protein. Nature, 414, 756-759.

13. Werner, T., Liu, G., Kang, D., Ekengren, S., Steiner, H. \& Hultmark, D. (2000). A family of peptidoglycan recognition proteins in the fruit fly Drosophila melanogaster. Proc. Natl Acad. Sci. USA, 97, 13772-13777.

14. Kabsch, W. \& Sander, C. (1983). Dictionary of protein secondary structure: pattern recognition of hydrogen-bonded and geometrical features. Biopolymers, 22, 2577-2637.

15. Holm, L. \& Sander, C. (1993). Protein structure comparison by alignment of distance matrices. J. Mol. Biol. 233, 123-136.

16. Pelton, J. G., Torchia, D. A., Meadow, N. D. \& Roseman, S. (1993). Tautomeric states of the activesite histidines of phosphorylated and unphosphorylated IIIGlc, a signal-transducing protein from
Escherichia coli, using two-dimensional heteronuclear NMR techniques. Protein Sci. 2, 543-558.

17. Petrosino, J. F., Pendleton, A. R., Weiner, J. H. \& Rosenberg, S. M. (2002). Chromosomal system for studying AmpC-mediated $\beta$-lactam resistance mutation in Escherichia coli. Antimicrob. Agents Chemother. 46, 1535-1539.

18. Stapleton, P., Shannon, K. \& Phillips, I. (1995). DNA sequence differences of ampD mutants of Citrobacter freundii. Antimicrob. Agents Chemother. 39, 2494-2498.

19. Liu, C., Xu, Z., Gupta, D. \& Dziarski, R. (2001). Peptidoglycan recognition proteins-a novel family of four human innate immunity pattern recognition molecules. J. Biol. Chem. 276, 34686-34694.

20. Liu, C., Gelius, E., Liu, G., Steiner, H. \& Dziarski, R. (2000). Mammalian peptidoglycan recognition protein binds peptidoglycan with high affinity, is expressed in neutrophils, and inhibits bacterial growth. J. Biol. Chem. 275, 24490-24499.

21. Tydell, C. C., Yount, N., Tran, D., Yuan, J. \& Selsted, M. E. (2002). Isolation, characterization, and antimicrobial properties of bovine oligosccharide-binding protein. J. Biol. Chem. 277, 19658-19664.

22. Rämet, M., Manfruelli, P., Pearson, A., MatheyPrevot, B. \& Ezekowitz, R. A. B. (2002). Functional genomic analysis of phagocytosis and identification of a Drosophila receptor for E. coli. Nature, 416, 644-648.

23. De Pauw, P., Neyt, C., Vanderwinkel, E., Wattiez, R. \& Falmagne, P. (1995). Characterization of human serum $N$-acetylmuramyl-L-alanine amidase purified by affinity chromatography. Protein Expr. Purif. 6, 371-378.

24. Wang, H. \& Zuiderweg, E. R. P. (1995). HCCHTOCSY spectroscopy of ${ }^{13} \mathrm{C}$-labelled proteins in $\mathrm{H}_{2} \mathrm{O}$ using heteronuclear cross-polarization and pulsedfield gradients. J. Biomol. NMR, 5, 207-211.

25. Ponstingl, H. \& Otting, G. (1998). Rapid measurement of scalar three-bond ${ }^{1} \mathrm{H}^{\mathrm{N}}-{ }^{1} \mathrm{H}^{\alpha}$ spin coupling constants in ${ }^{15} \mathrm{~N}$ labelled proteins. J. Biomol. NMR, 12, 319324

26. Bartels, C., Xia, T., Güntert, P., Billeter, M. \& Wüthrich, K. (1995). The program XEASY for computer-supported NMR spectral analysis. J. Biomol. NMR, 5, 1-10.

27. Güntert, P., Mumenthaler, C. \& Wüthrich, K. (1997). Torsion angle dynamics for NMR structure calculation with the new program DYANA. J. Mol. Biol. 273, 283-298.

28. Luginbühl, P., Güntert, P., Billeter, M. \& Wüthrich, K. (1996). The new program OPAL for molecular dynamics simulations and energy refinements of biological macromolecules. J. Biomol. NMR, 8, 136-146.

29. Laskowski, R. A., Rullmann, J. A. C., MacArthur, M. W., Kaptein, R. \& Thornton, J. M. (1996). AQUA and PROCHECK-NMR: programs for checking the quality of protein structures solved by NMR. J. Biomol. NMR, 8, 477-486.

30. Koradi, R., Billeter, M. \& Wüthrich, K. (1996). MOLMOL: a program for display and analysis of macromolecular structures. J. Mol. Graph. 14, 51-55.

31. Sevilla-Sierra, P., Otting, G. \& Wüthrich, K. (1993). Determination of the nuclear magnetic resonance structure of the DNA-binding domain of the P22 c2 repressor (1-76) in solution and comparison with 
the DNA-binding domain of the 434 repressor. J. Mol. Biol. 235, 1003-1020.

32. Dewar, M. J. S., Zoebisch, E. G. \& Healy, E. F. (1985).

Development and use of quantum mechanical molecular models. 76. AM1: a new general purpose quantum mechanical molecular model. J. Am. Chem. Soc. 107, 3902-3909.

33. Burkert, U. \& Allinger, N. L. (1982). Molecular Mechanics, American Chemical Society, Washington, DC.

Edited by M. F. Summers

(Received 28 October 2002; received in revised form 13 January 2003; accepted 16 January 2003)

Note added in proof: Peptidoglycan degrading $N$-acetylmuramoyl-L-alanine amidase activity was recently demonstrated experimentally for PGRP-SC1B (Mellroth, P., Karlsson, J. \& Steiner, H., J. Biol. Chem. 278, 7059-7064, 2003). 